\title{
Image database of Japanese food samples with nutrition information
}

\author{
Wataru Sato ${ }^{\text {Corresp., } 1}$, Kazusa Minemoto ${ }^{1}$, Reiko Sawada ${ }^{1}$, Yoshiko Miyazaki $^{2}$, Tohru Fushiki ${ }^{2}$ \\ ${ }^{1}$ Kyoto University, Kyoto, Japan \\ 2 Ryukoku University, Ohtsu, Japan \\ Corresponding Author: Wataru Sato \\ Email address: sato.wataru.4v@kyoto-u.ac.jp
}

Background. Visual processing of food plays an important role in controlling eating behaviors. Several studies have developed image databases of food to investigate visual food processing. However, few databases include non-Western foods and objective nutrition information on the foods. Methods. We developed an image database of Japanese food samples that has detailed nutrition information, including calorie, carbohydrate, fat, and protein contents. To validate the database, we presented the images, together with Western food images selected from an existing database, and had Japanese participants rate their affective (valence, arousal, liking, and wanting) and cognitive (naturalness, recognizability, and familiarity) appraisals and estimates of nutrition. Results. The results showed that all affective and cognitive appraisals (except arousal) of the Japanese food images were higher than those of Western food.

Correlational analyses found positive associations between the objective nutrition information and subjective estimates of the nutrition information, and between the objective calorie/fat content and affective appraisals. Conclusions. These data suggest that by using our image database, researchers can investigate the visual processing of Japanese food and the relationships between objective nutrition information and the psychological/neural processing of food. 
1 Running head: Image database of Japanese food samples

2

5 Wataru Sato ${ }^{1}$, Kazusa Minemoto ${ }^{1}$, Reiko Sawada ${ }^{1}$, Yoshiko Miyazaki², and Tohru

6 Fushiki²

7

$8{ }^{1}$ Kyoto University, Kyoto, Japan.

$9 \quad 2$ Ryukoku University, Ohtsu, Japan.

\section{Corresponding author}

12 Wataru Sato

1346 Shimoadachi, Sakyo, Kyoto, Kyoto, 606-8501, Japan.

14 E-mail adress: sato.wataru.4v@kyoto-u.ac.jp 


\section{Abstract}

17 Background. Visual processing of food plays an important role in controlling eating behaviors. Several studies have developed image databases of food to

19 investigate visual food processing. However, few databases include non-Western 20 foods and objective nutrition information on the foods.

21 Methods. We developed an image database of Japanese food samples that has 22 detailed nutrition information, including calorie, carbohydrate, fat, and protein 23 contents. To validate the database, we presented the images, together with 24 Western food images selected from an existing database, and had Japanese participants rate their affective (valence, arousal, liking, and wanting) and cognitive (naturalness, recognizability, and familiarity) appraisals and estimates of nutrition.

Results. The results showed that all affective and cognitive appraisals (except arousal) of the Japanese food images were higher than those of Western food. Correlational analyses found positive associations between the objective nutrition information and subjective estimates of the nutrition information, and between the objective calorie/fat content and affective appraisals.

Conclusions. These data suggest that by using our image database, researchers can investigate the visual processing of Japanese food and the relationships between objective nutrition information and the psychological/neural processing of food.

Keywords: arousal; Image database; Japanese food; liking; nutrition; valance; 
39 wanting. 


\section{Introduction}

Visual processing of food plays an important role in identifying inherent biologically-significant information from food, such as edibility (Tsourides et al., 2016). The sight of food elicits affective or hedonic responses (e.g., Rodríguez et al., 2005) that occur rapidly even before the conscious perception of food (Sato et al., 2016), which in turn motivates food intake (Yeomans, Blundell \& Leshem, 2004). Functional neuroimaging studies have shown that seeing food images and consuming taste solutions activate common neural circuits, including the gustatory and affective brain regions (e.g., Simmons et al., 2005; for reviews, see Huerta et al., 2014; van der Laan et al., 2011).

Studies have created standardized image databases of food to investigate visual food processing (Foroni et al., 2013; Blechert et al., 2014; Miccoli et al., 2014; Charbonnier et al., 2016). For example, Foroni et al. (2013) collected 252 images of natural or processed food, 43 images of rotten food, and 529 images of non-food materials in a web-based search and validated these stimuli by assessing the participants' appraisals, including valence, arousal, and familiarity. Their database was used in subsequent studies, such as a functional neuroimaging investigations of visual food processing (e.g., Mengotti, Foroni, \& Rumiati, 2019; Morys, Bode, \& Horstmann, 2018; Padulo et al., 2016).

However, two issues regarding a food image database remain. First, most of the existing databases contain only Western food. A number of cross-cultural psychological studies have shown that cultural aspects of food modulate hedonic appraisals while seeing and eating food (e.g., Wanich et al., 2018; Torrico et al., 
64 2019; Sato et al., 2019; for a review, see Prescott et al., 1998). For example,

65 Torrico et al. (2019) showed images of various food products to participants

66 with Western and Asian backgrounds and asked them to rate their preferences.

67 The food images from Western and Asian origins elicited stronger preferences in

68 the Western and Asian groups, respectively. These data suggest the need to

69 develop datasets of non-Western food images. To overcome this problem, a

70 recent study developed a food image database containing 209 images in the

71 Asian food category (Toet et al., 2019). It is desirable to develop non-Western

72 food image databases that have different specific advantages to investigate

73 visual food processing further.

74 Second, the existing databases do not provide nutrition information on the

75 foods. Although the total calories (Foroni et al., 2013; Charbonnier et al., 2016)

76 or the calories and carbohydrate, fat, and protein contents (Blechert et al., 2014)

77 in the materials were reported, the information was estimated using general data

78 and not the specific foods in the images (Foroni et al., 2013; Blechert et al.,

79 2014) or are not described in detail (Charbonnier et al., 2016; Toet et al., 2019).

80 This issue could be important, because it is widely believed that objective

81 nutrition information affects the visual processing of food (Birch, 1999) but

82 empirical data are scarce and mixed. Some studies provided positive evidence

83 that participants accurately estimated the total calories (Foroni et al., 2013;

84 Charbonnier et al., 2016; Brunstrom et al., 2018) and fat content (Toepel et al.,

85 2009) and that they showed a preference for high-calorie food (Brunstrom et al.,

86 2018) based on visual information on of the food materials. Other studies 
87 reported problematic estimates of total calories for food images (Carels, Konrad 88 \& Harper, 2007; Foroni, Pergola, \& Rumiati, 2016; Horne et al., 2019). To 89 investigate these relationships further, objective nutrition information is 90 indispensable for food image stimuli.

91 To investigate these issues, we developed an image database of Japanese 92 food samples (i.e., plastic replicas) (Figure 1). We photographed the stimuli 93 selected from a database of Japanese food samples that looked realistic, 94 included Japanese food that frequently appears in contemporary Japanese home 95 meals, and have detailed nutrition information (e.g., calorie, carbohydrate, fat, and protein contents). To validate the image database, we assessed the participants' subjective appraisals of their affective (valence, arousal, liking, and wanting) appraisals of the images. We also assessed cognitive appraisals to confirm whether food images looked as natural as food (naturalness) and were recognizable (recognizability) and familiar (familiarity). As reference data in relation to these appraisals, we additionally included images of Western food selected from an existing database (Blechert et al., 2014). We also assessed the subjective estimate of nutrition and investigated the relationships between objective nutrition information and the subjective perception of nutrition, as well as affective appraisals.

\section{Materials \& Methods}

\section{Participants}

The appraisal experiment enrolled 32 Japanese volunteers (13 females; mean \pm 
$110 S D$ age, $21.9 \pm 3.4$ years $)$. The required sample size was determined based on an

111 a priori power analysis using $\mathrm{G}^{*}$ Power software ver. 3.1.9.2 (Faul et al., 2007).

112 The $d$ of 0.5 (medium-size effect), an $\alpha$ level of 0.05 , and a power (1 - $\beta$ ) of

1130.80 were assumed in analyses of relationships between objective nutrition

114 information and subjective appraisals. The result of the power analysis showed

115 that more than 27 participants were needed. Participants were gathered through

116 advertising presented at the Kyoto University facility, and participants received

117 a book coupon corresponding to 500 Japanese yen. All participants were

118 confirmed not to have any food restrictions for medical or religious reasons. The

119 participants' hunger levels were rated on a 9-point scale from 1 (very hungry) to

1209 (very satiated) before the experiment, and the results showed that the majority

121 of them were in the neutral state (mean $\pm S D, 4.5 \pm 1.6)$. None of the

122 participants were obese (body mass index, $<30$, mean $\pm S D, 20.4 \pm 2.5 \mathrm{~kg} / \mathrm{m}^{2}$ ).

123 All participants had normal or corrected-to-normal visual acuity and had no

124 color vision deficiencies. After a detailed explanation of the experimental

125 procedure, all participants provided written informed consent. This study was

126 approved by the Ethics Committee of the Graduate School of Medicine, Kyoto

127 University (R0343), and was conducted in accordance with approved guidelines.

128 Apparatus

129 The experimental events were controlled by PowerPoint 2007 (Microsoft,

130 Redmond, WA, USA) implemented on a laptop computer (Precision M6300,

131 Dell, Round Rock, TX, USA).

132 Stimuli 
133 As Japanese food stimuli, we photographed items in a Japanese food sample 134 database, the Syokuiku Satisfactory "à La Carte” Tray (SAT) system (Iwasaki, 135 Osaka, Japan; http://www.foodmodel.com/category12/index.html). "Syokuiku" 136 is a Japanese term that means the education/promotion of food and nutrition. 137 The database contains 118 samples of Japanese foods that frequently appear in 138 contemporary Japanese home meals (e.g., meat and potato stew). The food 139 samples bear a close resemblance to actual food. Importantly, the SAT system 140 has nutrient information for each food item, including weight (g), calories 141 (kcal), moisture $(\mathrm{mL})$, carbohydrate $(\mathrm{g})$, fat $(\mathrm{g})$, and protein $(\mathrm{g})$, based on a 142 detailed analysis of materials included in the specific item (e.g., $7.5 \mathrm{~g}$ light soy 143 sauce in $\# 07$ Kitsune udon). We selected 46 processed food items (i.e., removing 144 non-processed food such as fruits) without obstacles (i.e., packaging and plastic 145 covers) and took color pictures using a digital camera (EXILIM FH100; Casio, 146 Tokyo, Japan). We selected only processed food items, because the SAT set had 147 a relatively small number of non-processed food items, and previous studies 148 have shown that affective appraisals can be different across processed vs. non149 processed food (Coricelli et al., 2019a). The pictures were then cropped and 150 modified to remove the background using Photoshop CS6 (Adobe, San Jose, CA, 151 USA).

152 For Western food stimuli, we selected five pictures of Western processed 153 food (e.g., meatballs; \#190, 315, 322, 324, and 384) from the existing database 154 (Blechert et al., 2014). We selected only five images because these were used as 155 the reference condition with the validated database images of actual food. We 
156 did not intend to compare thoroughly Western vs. Japanese food images.

157 Previous methodological studies suggested that three items would be needed to 158 represent a group reliably (MacCallum et al., 1999; Raubenheimer, 2004).

159 All stimuli measured horizontally $600 \times$ vertically 450 pixels. Some

160 examples of the Japanese food stimuli are shown in Figure 1 and all are shown

161 in Figure S1. The pictorialized Western food images are shown in Figure S2.

162 Procedure

163 To validate the image database of the Japanese food samples, we conducted a 164 appraisal experiment using the images of Japanese and Western food with a 165 paper-pencil questionnaire. The participants were tested individually. The 166 images were presented one at a time. Participants advanced the image by 167 themselves after appraising all items and they were forbidden to return to any 168 item. For each image, the participants were asked to rate valance (from 169 "negative" to "positive"), arousal (from "low arousal" to "high arousal"), and 170 liking, wanting, naturalness, recognizability, and familiarity (from "not at all" 171 to "very much") on 9-point Likert scales. The participants were also asked to 172 estimate the total calories (kcal) and caloric percentages of carbohydrate, fat, 173 and protein and describe the exact figures. In total, 51 trials (46 for Japanese 174 food; five for Western food) were performed. The order of trials was

175 randomized. At the beginning of the experiment, thumbnails of all images were 176 presented to enable participants to perceive all the images and rate them using a 177 wide range of scales (i.e., to reduce the anchor effect in Likert scales; Bishop \& 178 Herron, 2015), and then two practice trials were conducted using images not 
179 included in the database.

180 Data analysis

181 All statistical tests were performed using SPSS 16.0J software (SPSS Japan, 182 Tokyo, Japan). First, to compare the appraisals of Japanese and Western food 183 images, we calculated the mean appraisal for each image across participants.

184 Welch's $t$-tests (two-tailed) were conducted for appraisals (valence, arousal, 185 liking, wanting, naturalness, recognizability, and familiarity); Welch's $t$-tests 186 are more robust to unequal sample size (Delacre, Lakens, \& Leys, 2017). The 187 effect size $r$ (Cohen, 1992) was calculated.

188 Next, to evaluate the relationships between objective nutrition information 189 (calories (kcal) and relative caloric percentages of carbohydrate, fat, and 190 protein) and subjective nutrition/affective appraisals for the Japanese food 191 images, we calculated the Pearson's product-moment correlation coefficient 192 between the objective nutrition information and subjective appraisals across 193 images for each participant. Then, the correlation coefficients were normalized 194 using a Fisher's $r$-to- $z$ transformation and entered into Student's one-sample $t$ 195 tests to evaluate a significant difference from zero (two-tailed). We used this 196 two-stage random effect analyses to evaluate the generalizability of individual197 level statistical models, rather than calculating the correlations between 198 aggregated data (cf. Zhang \& Wang, 2014). However, to visualize heuristically 199 the relationships between objective nutrition information and subjective 200 appraisals, we depicted the scatterplots and regression lines using the 201 aggregated (group-mean) data in supplementary figures. In addition, we 
202 analyzed the relationships between the subjective estimates of nutrition

203 information and affective appraisals in the same way for descriptive purposes.

204 The results were considered statistically significant at $p<0.05$.

205 We conducted preliminary analyses of the factor sex and found no

206 significant effects on the results. Hence, this factor was disregarded. We also

207 performed preliminary analyses of the visual properties (brightness, spatial

208 frequency (i.e., overall activity level; Eskicioglu \& Fisher, 1995; Li et al.,

209 2001), and entropy (i.e., measure of randomness; Tsai, Lee \& Matsuyama,

210 2008)) of each Japanese food sample image using MATLAB 2018

211 (MathWorks, Natick, MA, USA). We confirmed that almost all of the reported

212 significant associations between the objective nutrition information and

213 subjective appraisals were significant even when the covariates (cf. Hedberg

$214 \&$ Ayers, 2015) of the correlation coefficients between visual properties and

215 subjective appraisals were included (File S1).

\section{Results}

218 Information of images

219 Figure 1 shows examples of images of the Japanese food samples and Table 1

220 lists the names and descriptions of all 46 items. Table 2 shows the mean (with

$221 S D$ ) subjective appraisals across all Japanese food samples. The mean (with $S D$ )

222 objective nutrition information of Japanese food samples came to: total calories,

$223236.7 \pm 194.1 \mathrm{kcal} ; \%$ carbohydrate, $34.99 \pm 25.6 ; \%$ fat, $38.1 \pm 20.8 ;$ and

$224 \%$ protein, $26.8 \pm 17.2$. 


\section{Subjective affective and cognitive appraisals}

226 Figure 2 shows the mean (with $S E$ ) subjective affective (valence, arousal, liking,

227 and wanting) and cognitive (naturalness, recognizability, and familiarity)

228 appraisals for each item. Welch's $t$-tests contrasting Japanese versus Western

229 food images showed significant differences for all measures $(t(49)>5.52, p<$

$2300.001, r>0.61$; Table 3), indicating higher appraisals for Japanese foods than

231 for Western foods, except the arousal appraisal $(t(49)=1.00, p>0.10, r=$

$2320.14)$. The data suggest that the images of Japanese food samples in the database

233 elicited stronger food-related positive affective appraisals (positive, liking, and

234 wanting), and looked more natural, recognizable, and familiar than the

235 photographs of Western food selected from the existing database (Blechert et

236 al., 2014).

237 Relationships between objective nutrition information and subjective food

238 appraisals

239 Next, we analyzed the relationships between the objective nutrition

240 information (calories, carbohydrate, fat, and protein) and subjective

241 nutrition/affective appraisals for food. Figure 3 shows the correlation

242 coefficients between the objective information and subjective estimates of

243 nutrition (cf. group-mean scatterplots with regression lines in Figure S3).

244 Student's one-sample $t$-tests after Fisher's $r$-to- $z$ transformation showed that

245 all of the relationships were positive and significant $(t(31)>11.32, p<$

$2460.001, r>0.53$; Table 4).

247 Figure 4 shows the correlation coefficients between the objective 
248 information and affective appraisals (cf. group-mean scatterplots with

249 regression lines in Figure S4). Student's one-sample $t$-tests after Fisher's $r$-to- $z$

250 transformation for objective information showed that objective calories and fat

251 were significantly and positively correlated with all of the affective food

252 appraisals $(t(31)>2.70, p<0.05, r>0.37$; Table 5). Significant negative

253 correlations were detected between objective carbohydrate and valence,

254 liking, and wanting $(t(31)>2.72, p<0.05, r>0.42)$ and between objective

255 protein and arousal $(t(31)=6.02, p<0.001, r=0.69)$.

256 In addition, we exploratorily analyzed the relationships between the

257 subjective estimates of nutrition information and affective appraisals (Figure

$2584)$. The analysis showed the same significant patterns as the results of

259 objective information for subjective calories and fat $(t(31)>2.70, p<0.05, r$

$260>0.37$; Table 5), except that the subjective fat-valence relationship did not

261 reach significance $(t(31)=1.57, p>0.10, r=0.26)$. Subjective carbohydrate

262 and protein showed rather different patterns, including a significant positive

263 correlation between subjective carbohydrate and arousal $(t(31)=2.27, p<$

$2640.05, r=0.36$ ) and negative correlations between subjective protein and

265 arousal, liking, and wanting $(t(31)>2.09, p<0.05, r>0.33)$.

267 Discussion

268 Our data on the subjective affective appraisals showed that the images of

269 Japanese food samples were more positive, liked, and wanted by Japanese

270 participants than the five selected images of Western food items. The

271 naturalness appraisals showed that the images of Japanese food samples were 
272 rated more natural than the Western food images selected from the existing

273 database. This result suggests that the food sample images looked almost as real

274 as actual food. The results for familiarity and recognizability showed that the

275 Japanese foods in the database were more familiar and more easily recognizable

276 by Japanese participants than the selected Western food items. Overall, these

277 results indicate that the images of Japanese food samples we developed validly

278 represent Japan foods.

279 Because our food image database had objective nutrition information, we

280 analyzed the relationships between objective nutrient information and subjective

281 nutrition and affective appraisals. The results showed that the objective

282 information and subjective perception of nutrition were positively associated,

283 and that the objective caloric content and percentage of fat calories were

284 positively associated with all of the affective appraisals. These results are

285 consistent with previous findings that participants estimated the caloric (e.g.,

286 Foroni et al., 2013) and fat (Toepel et al., 2009) contents precisely and reported

287 a greater liking for food with high caloric values (Brunstrom et al., 2018) during

288 visual food processing, although other studies failed to find such patterns (e.g.,

289 Carels et al., 2007) and debate remains. However, previous studies estimated the

290 nutrition information using a general database and did not investigate the

291 contents of specific food images. The previous studies also did not investigate

292 systematically the carbohydrate, fat, and protein contents. Therefore, we

293 investigated this and found that the relative caloric ratio of carbohydrate and

294 protein was negatively associated with the affective appraisals of food. Our 
295 results also showed that the relationships between objective nutrition

296 information and affective appraisals are rather similar to those between

297 subjective nutrition perception and affective appraisals in terms of caloric and

298 fat contents, but not in terms of carbohydrate and protein. In summary, our

299 results confirm and extend previous findings indicating that objective nutrition

300 information about food can influence the subjective estimates of nutrition and

301 affective appraisals during the visual processing of food.

302 The database of Japanese food images with nutrition information that we 303 developed has practical significance for research on visual food processing. For

304 example, it would be interesting to use our database in functional neuroimaging

305 studies. Previous studies have shown that several brain regions, including the

306 visual cortices (e.g., the fusiform gyrus) and limbic regions (e.g., the

307 amygdala), are activated more during the presentation of food images than non-

308 food images (e.g., Holsen et al., 2005; Sato et al., 2019; for a review, see van

309 Meer et al., 2015). Although a few studies compared neural activity in response

310 to low vs high calorie/fat food (Frank et al., 2010; Killgore et al., 2003; Toepel

311 et al., 2009), more detailed parametric relationships between neural activity and 312 objective nutrition information remain unexplored. Use of the current database

313 may allow studies of the specific brain activities associated with objective 314 nutrition.

315 Several limitations of this study should be acknowledged. First, our 316 database was restricted to a small number of food samples. Therefore, the 317 number of images should be increased. Developing a comparable image database 
318 using real food with detailed nutrition information may also be helpful. Second,

319 our stimuli were restricted to processed food. Because some previous studies

320 have suggested different psychological (Aiello et al., 2018; Coricelli et al.,

321 2019a; Rumiati et al., 2016) and neural (Coricelli et al., 2019b; Pergola et al.,

322 2017) processing between processed and non-processed food, the

323 generalizability of the current results for non-processed food is an important

324 matter for future research. Third, our food stimuli were depicted in different

325 plates. This was because the Japanese diet has custom rules for plates

326 (Thompson, 2016) and the food sample set we photographed mimicked this to

327 create realistic Japanese food stimuli. This may be problematic, because some

328 previous studies have shown that plates can influence the affective response to

329 food items (Piqueras-Fiszman, et al., 2012; Piqueras-Fiszman, Giboreau, \&

330 Spence, 2013; Stewart \& Goss, 2013; Van Ittersum \& Wansink, 2012). An

331 assessment using the present images after removing the plates is warranted to

332 confirm the findings. Fourth, we assessed only subjective appraisals. Because

333 subjective appraisals could be biased to the results that researchers want to find

334 due to participants' care about demand characteristics (Orne, 1962), objective

335 measures of affective responses, such as physiological signals (Kaneko et al.,

336 2018), may complement the current findings. Finally, we tested only a small

337 sample of participants. We determined the sample size to detect more than

338 middle size effects (cf. Cohen, 1992). Because our sample included only young

339 participants, it remains unknown whether participants of different age groups,

340 such as children and older participants, would show similar affective and 
341 cognitive appraisals, and concordance between objective nutrition information

342 and subjective appraisals. We tested only Japanese participants; hence, the

343 patterns may be different in different cultures. We also did not assess the details

344 of participants' characteristics that could modulate food processing, such as

345 dieting habits (Coricelli et al., 2019a; Hoefling \& Strack, 2008). Investigations

346 including more participants from different age groups and different cultures,

347 with detailed assessments of their characteristics, by using the present

348 stimulus database would be valuable for investigating visual food processing

349 further.

350

\section{Conclusions}

352 We developed an image database of Japanese food samples that contains

353 detailed nutrition information, including calorie, carbohydrate, fat, and protein

354 contents. The appraisal experiments showed that all affective and cognitive

355 appraisals of the Japanese food sample images were higher than, or comparable

356 to, those of Western foods. Correlational analyses showed positive associations

357 between the objective information and subjective perception of the calorie,

358 carbohydrate, fat, and protein contents, and objective calorie/fat content and

359 affective appraisals. These data suggest that by using our image database,

360 researchers can investigate the visual processing of Japanese food and

361 relationships between objective nutrition information and psychological/neural

362 processing of food. This image database is available on request from the

363 corresponding author solely for research purposes. The nutrition information for 
364 the stimuli is available from the Iwasaki SAT system

365 (http://www.foodmodel.com/category 12/index.html). 


\section{Acknowledgements}

368 The authors would like to thank Dr. Hiromi Hata and Ms. Yukari Sato for their

369 technical support.

370

371 Funding

372 This study was supported by grants from the Project of the NARO Bio-oriented

373 Technology Research Advancement Institution (Integration Research for

374 Agriculture and Interdisciplinary Fields, Japan), the Research Complex Program

375 from Japan Science and Technology Agency, and Japan Society for the Promotion

376 of Science KAKENHI (18K03174).

377

378 Competing Interests

379 The authors declare there are no competing interests. The authors declare no 380 competing financial or other interests.

\section{Author Contributions}

383 - Wataru Sato conceived and designed the experiments, performed the $\square$ 384 experiments, analyzed the data, wrote the paper.

385 - Kazusa Minemoto performed the experiments, wrote the paper.

386 - Reiko Sawada performed the experiments, wrote the paper.

387 - Yoshiko Miyazaki performed the experiments.

388 - Tohru Fushiki conceived and designed the experiments, performed the $\square$ 389 experiments, wrote the paper. 


\section{Human Ethics}

392 The study was approved by the Ethics Committee of the Graduate School of 393 Medicine, Kyoto University (No. R0256).

394

395 Data Availability

396 The following information was supplied regarding data availability:

397 Raw data is available in the Supplemental Files. 
399

400

401

402

403

404

405

406

407

408

409

410

411

412

413

414

415

416

417

418

419

420

421

\section{References}

Aiello M, Vignando M, Foroni F, Pergola G, Rossi P, Silveri MC, Rumiati RI. 2018. Episodic memory for natural and transformed food. Cortex 107: 13-20.

Birch LL. 1999. Development of food preferences. Annual Review of Nutrition 19:41-62.

Bishop PA, Herron RL. 2015. Use and misuse of the Likert item responses and other ordinal measures. International Journal of Exercise Science $8: 297-302$.

Blechert J, Meule A, Busch NA, Ohla K. 2014. Food-pics: an image database for experimental research on eating and appetite. Frontiers in Psychology 5:617.

Brunstrom JM, Drake ACL, Forde CG, Rogers PJ. 2018. Undervalued and ignored: Are humans poorly adapted to energy-dense foods? Appetite $120: 589-595$.

Carels RA, Konrad K, Harper J. 2007. Individual differences in food perceptions and calorie estimation: an examination of dieting status, weight, and gender. Appetite 49:450-458.

Cohen, J. 1992. A power primer. Psychological Bulletin 112:155-159.

Coricelli C, Foroni F, Osimo SA, Rumiati RI. 2019a. Implicit and explicit evaluations of foods: The natural and transformed dimension. Food quality and preference 73: 143-153.

Coricelli C, Toepel U, Notter ML, Murray MM, Rumiati RI. 2019b. Distinct 
422

424 425

426

427

428

429

430

431

432

433

434

435

436

437

438

439

440

441

442

443

444

brain representations of processed and unprocessed foods. European Journal of Neuroscience 50: 3389-3401.

Charbonnier L, van Meer F, van der Laan LN, Viergever MA, Smeets PAM. 2016. Standardized food images: A photographing protocol and image database. Appetite 96:166-173.

Delacre M, Lakens D, Leys C. 2017. Why psychologists should by default use Welch's t-test instead of Student's t-test. International Review of Social Psychology 30:92-101.

Eskicioglu AM, Fisher PS. 1995. Image quality measures and their performance. IEEE Transactions on Communications 43:2959-2965.

Faul F, Erdfelder E, Lang AG, Buchner A. 2007. G*Power 3: A flexible statistical power analysis program for the social, behavioral, and biomedical sciences. Behavior Research Methods 39:175-191.

Foroni F, Pergola G, Argiris G, Rumiati RI. 2013. The FoodCast research image database (FRIDa). Frontiers in Human Neuroscience 7:51.

Foroni F, Pergola G, Rumiati RI. 2016. Food color is in the eye of the beholder: The role of human trichromatic vision in food evaluation. Scientific Reports 6, 37034 .

Frank S, Laharnar N, Kullmann S, Veit R, Canova C, Hegner YL, Fritsche A, Preissl H. 2010. Processing of food pictures: influence of hunger, gender and calorie content. Brain Research 1350:159-166.

Hedberg EC, Ayers S. 2015. The power of a paired t-test with a covariate. Social Science Research 50:277-291. 
445 Hoefling A, Strack F. 2008. The tempting effect of forbidden foods. High

446

447

448

449

450

451

452

453

454

455

456

457

458

459

460

461

462

463

464

465

466

467

calorie content evokes conflicting implicit and explicit evaluations in restrained eaters. Appetite 51:681-689.

Holsen LM, Zarcone JR, Thompson TI, Brooks WM, Anderson MF, Ahluwalia JS, Nollen NL, Savage CR. 2005. Neural mechanisms underlying food motivation in children and adolescents. Neuroimage 27:669-676.

Horne D, Palermo R, Neumann MF, Housley R, Bell J. 2019. Can People Accurately Estimate the Calories in Food Images? An Optimised Set of Low- and High-Calorie Images from the food-pics database. Appetite 139:189-196.

Huerta CI, Sarkar PR, Duong TQ, Laird AR, Fox PT. 2014. Neural bases of food perception: coordinate-based meta-analyses of neuroimaging studies in multiple modalities. Obesity 22:1439-1446.

Kaneko D, Toet A, Brouwer AM, Kallen V, van Erp JBF. 2018. Methods for evaluating emotions evoked by food experiences: A literature review. Frontiers in Psychology 9:911.

Killgore WD, Young AD, Femia LA, Bogorodzki P, Rogowska J, YurgelunTodd DA. 2003. Cortical and limbic activation during viewing of highversus low-calorie foods. Neuroimage 19:1381-1394.

Li S, Kwok JT, Wang Y. 2001. Combination of images with diverse focuses using the spatial frequency. Information Fusion 2:169-176.

MacCallum RC, Widaman KF, Zhang S, Hong S. 1999. Sample size in factor analysis. Psychological Methods 4:84-99. 
468 Mengotti P, Foroni F, Rumiati RI. 2018. Neural correlates of the energetic 469 value of food during visual processing and response inhibition. 470 Neuroimage 184:130-139.

471 Miccoli L, Delgado R, Rodríguez-Ruiz S, Guerra P, García-Mármol E, 472 Fernández-Santaella MC. 2014. Meet OLAF, a good friend of the IAPS!

473

474

475

476

477

478

479

480

481

482

483

484

485

486

487

488

489

490 The Open Library of Affective Foods: a tool to investigate the emotional impact of food in adolescents. PLoS One 9:e114515.

Morys F, Bode S, Horstmann A. 2018. Dorsolateral and medial prefrontal cortex mediate the influence of incidental priming on economic decision making in obesity. Scientific Reports 8:17595.

Orne MT. 1962. On the social psychology of the psychological experiment: With particular reference to demand characteristics and their implications. American Psychologist 17:776-783.

Padulo C, Delli Pizzi S, Bonanni L, Edden RA, Ferretti A, Marzoli D, Franciotti R, Manippa V, Onofrj M, Sepede G, Tartaro A, Tommasi L, PuglisiAllegra S, Brancucci A. 2016. GABA levels in the ventromedial prefrontal cortex during the viewing of appetitive and disgusting food images. Neuroscience 333:114-122.

Pergola G, Foroni F, Mengotti P, Argiris A, Rumiati RI. 2017. A neural signature of food semantics is associated with body-mass index. Biological Psychology 129:282-292.

Piqueras-Fiszman B, Alcaide J, Roura E, Spence C. 2012. Is it the plate or is it the food? Assessing the influence of the color (black or white) and 
491

492

\section{3}

494

495

496

497

498

499

500

501

502

503

504

505

506

507

508

509

510

511

shape of the plate on the perception of the food placed on it. Food Quality and Preference 24:205-208.

Piqueras-Fiszman B, Giboreau A, Spence C. 2013. Assessing the influence of the color of the plate on the perception of a complex food in a restaurant setting. Flavour $2: 24$.

Prescott J, Bell GA, Gillmore R, Yoshida M, O'Sullivand M, Korac S, Allen S, Yamazaki K. 1998. Cross-cultural comparisons of Japanese and Australian responses to manipulations of sourness, saltiness and bitterness in foods. Food Quality and Preference 9:53-66.

Raubenheimer J. 2004. An item selection procedure to maximise scale reliability and validity. SA Journal of Industrial Psychology 30: 59-64.

Rodríguez S, Fernández MC, Cepeda-Benito A, Vila J. 2005. Subjective and physiological reactivity to chocolate images in high and low chocolate cravers. Biological Psychology 70:9-18.

Rumiati RI, Foroni F, Pergola G, Rossi P, Silveri MC. 2016. Lexical-semantic deficits in processing food and nonfood items. Brain and Cognition $110: 120-130$.

Sato W, Kochiyama T, Minemoto K, Sawada R, Fushiki T. 2019. Amygdala activation during unconscious visual processing of food. Scientific Reports 9:7277.

Sato W, Rymarczyk K, Minemoto K, Wojciechowski J, Hyniewska S. 2019. Cultural modulation of unconscious hedonic responses to food. Nutrients 11:2832. 
514 Sato W, Sawada R, Kubota Y, Toichi M, Fushiki T. 2016. Unconscious affective responses to food. PLoS One 11:e0160956.

516 Simmons WK, Martin A, Barsalou LW. 2005. Pictures of appetizing foods activate gustatory cortices for taste and reward. Cerebral Cortex $15: 1602-1608$.

Stewart PC, Goss E. 2013. Plate shape and colour interact to influence taste and quality judgments. Flavour 2:27.

521 Thompson, J. 2016. The art of Japanese plating. Metropolis (https://metropolisjapan.com/art-japanese-plating/).

523 Toepel U, Knebel JF, Hudry J, le Coutre J, Murray MM. 2009. The brain tracks the energetic value in food images. Neuroimage 44:967-974.

525 Toet A, Kaneko D, de Kruijf I, Ushiama S, van Schaik MG, Brouwer AM, Kallen V, van Erp JBF. 2019. CROCUFID: A cross-cultural food image database for research on food elicited affective responses. Frontiers in Psychology 10:58.

Torrico DD, Fuentes S, Gonzalez Viejo C, Ashman H, Dunshea FR. 2019. Cross-cultural effects of food product familiarity on sensory

Tsourides K, Shariat S, Nejati H, Gandhi TK, Cardinaux A, Simons CT, Cheung NM, Pavlovic V, Sinha P. 2016. Neural correlates of the food/non-food acceptability and non-invasive physiological responses of consumers. Food Research International 115:439-450.

536 Tsai DY, Lee Y, Matsuyama E. 2008. Information entropy measure for 
evaluation of image quality. Journal of Digital Imaging 21:338-347.

538 van der Laan LN, de Ridder DT, Viergever MA, Smeets PA. 2011. The first taste is always with the eyes: a meta-analysis on the neural correlates of processing visual food cues. NeuroImage 55:296-303.

Van Ittersum K, Wansink B. 2012. Plate size and color suggestibility: The Delboeuf Illusion's bias on serving and eating behavior. Journal of Consumer Research 39:215-228.

544 van Meer F, van der Laan LN, Adan RA, Viergever MA, Smeets PA. 2015. What 545 you see is what you eat: an ALE meta-analysis of the neural correlates of food viewing in children and adolescents. Neuroimage 104:35-43.

547 Wanich U, Sayompark D, Riddell L, Cicerale S, Liem DG, Mohebbi M, 548

Yeomans MR, Blundell JE Food Liking Questionnaires and direct food tasting in two cultures. nutritional need or need-free stimulation of appetite? The British Journal of Nutrition 92 Supp1 1:S3-14.

554 Zhang QJ, Wang LP. 2014. Aggregating and testing intra-individual 555 correlations: Methods and comparisons. Multivariate Behavioral Research 49: 130-148. 


\section{Figure legends}

559 Figure 1. Examples of food stimuli. The items \#07 (Kitsune udon; A), \#10 (Sake 560 no shioyaki; B), and \#26 (Tonkatsu; C) are shown.

561

562 Figure 2. Mean (with standard error) subjective appraisals of Japanese food 563 sample images and Western food images. Affective (valence (A), arousal (B), 564 liking (C), and wanting (D)) and cognitive (naturalness (E), recognizability (F), 565 and familiarity $(\mathrm{G}))$ appraisals are shown. Numbers on the $x$-axes are the items 566 in Table $1 . * * *, p<0.001$ ( $t$-tests).

567

568 Figure 3. Mean (with standard error) intra-individual correlation coefficients 569 between objective and subjective nutrition information for Japanese food sample 570 images. $* * *, p<0.001$ (one-sample $t$-tests after Fisher's transformation).

572 Figure 4. Mean (with standard error) intra-individual correlation coefficients 573 between objective/subjective nutrition information and affective appraisals for 574 Japanese food sample images. The correlations of affective appraisals with 575 objective calorie (A), objective carbohydrate (B), objective fat (C), objective 576 protein (D), subjective calorie (E), subjective carbohydrate (F), subjective fat 577 (G), and subjective protein $(\mathrm{H})$ are shown. ${ }^{* * *}, p<0.001 ; * *, p<0.01 ; *, p<$ 578 0.05 (one-sample $t$-tests after Fisher's transformation).

580 Figure S1. All Japanese food sample images. 
582 Figure S2. Pictorialized Western food images.

583

584 Figure S3. Group-mean (aggregated) scatterplots and regression lines of the 585 relationships between objective nutrition information (calories (kcal) and 586 relative caloric percentages of carbohydrate, fat, and protein) and subjective 587 nutrition appraisals.

588

589 Figure S4. Group-mean (aggregated) scatterplots and regression lines of the 590 relationships between objective/subjective nutrition information (calories (kcal)

591 and relative caloric percentages of carbohydrate, fat, and protein) and subjective 592 affective appraisals (valence, arousal, liking, and wanting). 


\section{Table 1 (on next page)}

Table 1 Namesand descriptions of the food items.

The names of the Japanese food are in Japanese. Numbers with the Western food descriptions refer to the item numbers in the database ( Blechert et al., 2014) . 
1 Table 1. Names and descriptions of the food items.

\begin{tabular}{rll}
\hline ID & Name & Description \\
\hline Japanese & \\
01 & Oyakodon & A bowl of rice with chicken, egg, and vegetables \\
02 & Aij no hiraki & Grilled sun-dried horse mackerel \\
03 & Tempura moriawase & Deep-fried fish and vegetables in a light batter \\
04 & Nikujaga & Meat and potato stew \\
05 & Chikuzenni & Chicken stew with vegetables \\
06 & Buri no teriyaki & Grilled teriyaki flavored yellowtail \\
07 & Kitsune udon & Udon noodles with deep-fried tofu \\
08 & Nigiri sushi & Hand-formed sushi \\
09 & Hiyayakko & Cold tofu \\
10 & Sake no shioyaki & Grilled salmon with salt \\
11 & Natto & Fermented soybeans \\
12 & Dashimaki & Rolled Japanese-style omelet \\
13 & Saba no nitsuke & Simmered mackerel \\
14 & Kabocha no nimono & Boiled pumpkin \\
15 & Yasai no nimono & Boiled vegetables \\
16 & U no hana & Bean curd dregs \\
17 & Gomoku nimame & Boiled beans \\
18 & Komatsuna no ohitashi & Soaked Japanese mustard spinach \\
19 & Kimpira gobo & Kimpira-style sautéed burdock \\
20 & Kiribothi daikon no nimono & Stewed dried radish \\
21 & Sirloin steak & Sirloin steak \\
22 & Buta no shogayaki & Ginger-fried pork \\
23 & Tori no karage & Fried chicken \\
24 & Wakame to kyuri no tsukemono & Vinegared Wakame seaweed and cucumber \\
25 & Mix fry & Assorted breaded deep-fried food \\
26 & Tonkatsu & Pork cutlet \\
27 & Butanikuiri yasaiitame & Fried vegetables with pork \\
28 & Hamburg & Hamburg steak \\
29 & Katsuo no tataki & Lightly roasted bonito \\
30 & Spaghetti meat sauce & Spaghetti with meat sauce \\
31 & Curry and rice & Curry and rice \\
32 & Sanma no shioyaki & Saury grilling fish with salt \\
33 & Plain omelet & Plain omelet \\
34 & Sashimi moriawase & Assorted sliced raw fish \\
35 & Takoyaki & Octopus dumplings \\
36 & Set yakimeshi & Fried rice \\
37 & Gyoza & Gyoza dumplings \\
38 & Chawanmushi & A savory steamed egg custard with assorted ingredients \\
39 & Koyadohu no nimono & Boiled freeze-dried tofu \\
& &
\end{tabular}


40 Oden

41 Tamagodofu

42 Yasai no misoshiru

43 Asari no sumashijiru

44 Anpan

45 Potate fri

46 Doughnut

\section{Western}

01 Frikadellen

02 Shashlik

03 Knuckle of pork with sauerkraut

04 Rissole

05 Tortellini
Vegetables, fish dumplings and various other stewed items

Steamed egg custard

Miso soup with vegetables

Clear soup with clams

A sweet roll filled with red bean paste

French fried potatoes

A doughnut

Flat, pan-fried meatballs of minced meat (\#190)

Skewered and grilled cubes of meat (\#315)

Leg of pork with sauerkraut (\#322)

A small fried ball of chopped meat or vegetables (\#324)

Ring-shaped pasta (\#384)

2

3 The names of the Japanese food are in Japanese. Numbers with the Western

4 food descriptions refer to the item numbers in the database (Blechert et al., $52014)$.

6 


\section{Table 2 (on next page)}

\section{Table 2}

Mean (with standard deviation) subjective appraisals for all Japanese foodsample images.

Affective and cognitive ratings (valence - familiarity) are on a 9-point scale of 1-9. Calorie is in kcal, and carbohydrate, fat, protein are in $\%$. 
1 Table 2. Mean (with standard deviation) subjective appraisals for all Japanese food sample images.

\begin{tabular}{cccccccccccc}
\hline Valence & Arousal & Liking & Wanting & Naturalness & Recognizability & Familiarity & Calorie & Carbohydrate & Fat & Protein \\
\hline 5.9 & 5.5 & 6.1 & 5.5 & 7.7 & 5.6 & 5.9 & 242.2 & 33.1 & 18.9 & 48.0 \\
$(0.6)$ & $(0.9)$ & $(0.8)$ & $(0.8)$ & $(0.8)$ & $(0.8)$ & $(1.0)$ & $(127.7)$ & $(16.8)$ & $(8.5)$ & $(17.3)$ \\
\hline
\end{tabular}

2

3 Affective and cognitive appraisals (valence-familiarity) are in 9-point scale in 1-9. Calorie is in kcal, and 4 carbohydrate, fat, protein are in $\%$. 


\section{Table 3 (on next page)}

Table 3 Results of Welch's t-tests (two-tailed) contrasting subjective appraisals for Japanese versus Western food images.

Degrees of freedom were $4.42-5.87$. Significant results $(p<0.05)$ are in bold. 
1 Table 3. Results of Welch's $t$-tests (two-tailed) contrasting subjective appraisals

2 for Japanese versus Western food images.

\begin{tabular}{cccccccc}
\hline Statistic & Valence & Arousal & Liking & Wanting & Naturalness & Recognizability & Familiarity \\
\hline$t$ & $\mathbf{5 . 7 6}$ & 1.21 & $\mathbf{5 . 3 5}$ & $\mathbf{6 . 4 5}$ & $\mathbf{9 . 2 9}$ & $\mathbf{8 . 3 4}$ & $\mathbf{7 . 9 5}$ \\
$p$ & $\mathbf{0 . 0 0 3}$ & 0.277 & $\mathbf{0 . 0 0 4}$ & $\mathbf{0 . 0 0 1}$ & $\mathbf{0 . 0 0 0}$ & $\mathbf{0 . 0 0 0}$ & $\mathbf{0 . 0 0 0}$ \\
$d$ & $\mathbf{2 . 5 4}$ & 0.41 & $\mathbf{2 . 1 7}$ & $\mathbf{2 . 2 2}$ & $\mathbf{4 . 3 2}$ & $\mathbf{3 . 3 1}$ & $\mathbf{2 . 4 9}$ \\
\hline
\end{tabular}

3

4 Degrees of freedom were 4.42-5.87. Significant results $(p<0.05)$ are in bold. 


\section{Table 4 (on next page)}

Table 4 Results of Student's one-sample t-tests (two-tailed) for the correlation coefficients between the objective information and subjective estimation of nutrition.

Data were analyzed after Fisher's $r$-to- $z$ transformation. Degrees of freedom were 31 . Significant results ( $p$ $<0.05)$ are in bold. 
1 Table 4. Results of Student's one-sample $t$-tests (two-tailed) for the

2 correlation coefficients between the objective information and subjective

3 estimation of nutrition.

\begin{tabular}{ccccc}
\hline Statistic & Calories & Carbohydrate & Fat & Protein \\
\hline$t$ & $\mathbf{2 5 . 6 2}$ & $\mathbf{1 2 . 7 2}$ & $\mathbf{1 1 . 3 2}$ & $\mathbf{1 5 . 0 4}$ \\
$p$ & $\mathbf{0 . 0 0 0}$ & $\mathbf{0 . 0 0 0}$ & $\mathbf{0 . 0 0 0}$ & $\mathbf{0 . 0 0 0}$ \\
$d$ & $\mathbf{4 . 5 3}$ & $\mathbf{2 . 2 5}$ & $\mathbf{2 . 0 0}$ & $\mathbf{2 . 6 6}$ \\
\hline
\end{tabular}

4

5 Data were analyzed after Fisher's $r$-to- $z$ transformation. Degrees of freedom

6 were 31 . Significant results $(p<0.05)$ are in bold. 


\section{Table 5 (on next page)}

Table 5 Results of Student's one-sample t-tests (two-tailed) for the correlation coefficients between the objective/subjective nutrition and affective appraisals.

Data were analyzed after Fisher's $r$-to- $z$ transformation. Degrees of freedom were 31 . Significant results ( $p$ $<0.05)$ are in bold. 
1 Table 5. Results of Student's one-sample $t$-tests (two-tailed) for the

2 correlation coefficients between the objective/subjective nutrition and

3 affective appraisals.

\begin{tabular}{|c|c|c|c|c|c|}
\hline \multirow[t]{2}{*}{ Nutrition } & \multirow[t]{2}{*}{ Statistic } & \multicolumn{4}{|c|}{ Affective appraisal } \\
\hline & & Valence & Arousal & Liking & Wanting \\
\hline \multirow[t]{3}{*}{ Objective calorie } & $t$ & 2.34 & 9.28 & 6.02 & 3.46 \\
\hline & $p$ & 0.025 & 0.000 & 0.000 & 0.001 \\
\hline & $d$ & 0.37 & 1.61 & 1.01 & 0.56 \\
\hline \multirow[t]{3}{*}{ Objective carbohydrate } & $t$ & 2.62 & 0.98 & 2.67 & 2.28 \\
\hline & $p$ & 0.013 & 0.336 & 0.012 & 0.029 \\
\hline & $d$ & 0.50 & 0.21 & 0.51 & 0.44 \\
\hline \multirow[t]{3}{*}{ Objective fat } & $t$ & 2.99 & 6.03 & 5.05 & 3.29 \\
\hline & $p$ & 0.005 & 0.000 & 0.000 & 0.002 \\
\hline & $d$ & 0.48 & 1.01 & 0.84 & 0.53 \\
\hline \multirow[t]{3}{*}{ Objective protein } & $t$ & 0.84 & 5.53 & -1.11 & 0.27 \\
\hline & $p$ & 0.409 & 0.000 & 0.274 & 0.793 \\
\hline & $d$ & 0.11 & 1.06 & 0.23 & 0.01 \\
\hline \multirow[t]{3}{*}{ Subjective calories } & $t$ & 2.73 & 9.03 & 6.73 & 4.29 \\
\hline & $p$ & 0.010 & 0.000 & 0.000 & 0.000 \\
\hline & $d$ & 0.48 & 1.60 & 1.19 & 0.76 \\
\hline \multirow[t]{3}{*}{ Subjective carbohydrate } & $t$ & 0.26 & 2.27 & 1.03 & 0.75 \\
\hline & $p$ & 0.799 & 0.030 & 0.313 & 0.459 \\
\hline & $d$ & 0.05 & 0.40 & 0.18 & 0.13 \\
\hline \multirow[t]{3}{*}{ Subjective fat } & $t$ & 1.57 & 6.06 & 4.80 & 3.24 \\
\hline & $p$ & 0.127 & 0.000 & 0.000 & 0.003 \\
\hline & $d$ & 0.28 & 1.07 & 0.85 & 0.57 \\
\hline \multirow[t]{3}{*}{ Subjective protein } & $t$ & 0.96 & 5.98 & 3.44 & 2.10 \\
\hline & $p$ & 0.342 & 0.000 & 0.002 & 0.044 \\
\hline & $d$ & 0.17 & 1.06 & 0.61 & 0.37 \\
\hline
\end{tabular}

4

5 Data were analyzed after Fisher's $r$-to-z transformation. Degrees of freedom

6 were 31 . Significant results $(p<0.05)$ are in bold. 
Figure 1

Figure 1 Examples of food stimuli.

The items \#07 (Kitsune udon; A), \#10 (Sake no shioyaki; B), and \#26 (Tonkatsu; C) are shown.
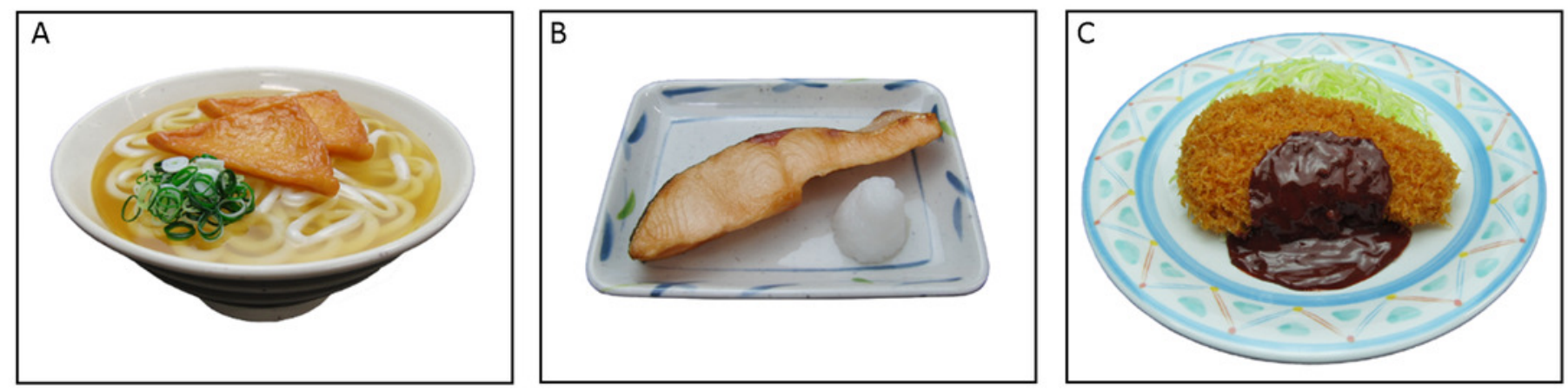


\section{Figure 2}

Figure 2

Mean (with standard error) subjective ratings of affective andcognitive appraisals of Japanese food sample images and Western food images.

Numbers on the $x$-axes are the items in Table 1. ${ }^{* * *}, p<0.001$ ( $t$-tests ).Affective (valence $(A)$, arousal (B), liking (C), and wanting (D)) and cognitive (naturalness (E), recognizability (F), and familiarity $(\mathrm{G})$ ) appraisals are shown. Numbers on the $\mathrm{x}$-axes are the items in Table 1. $* * *, p<0.001$ (t-tests). 


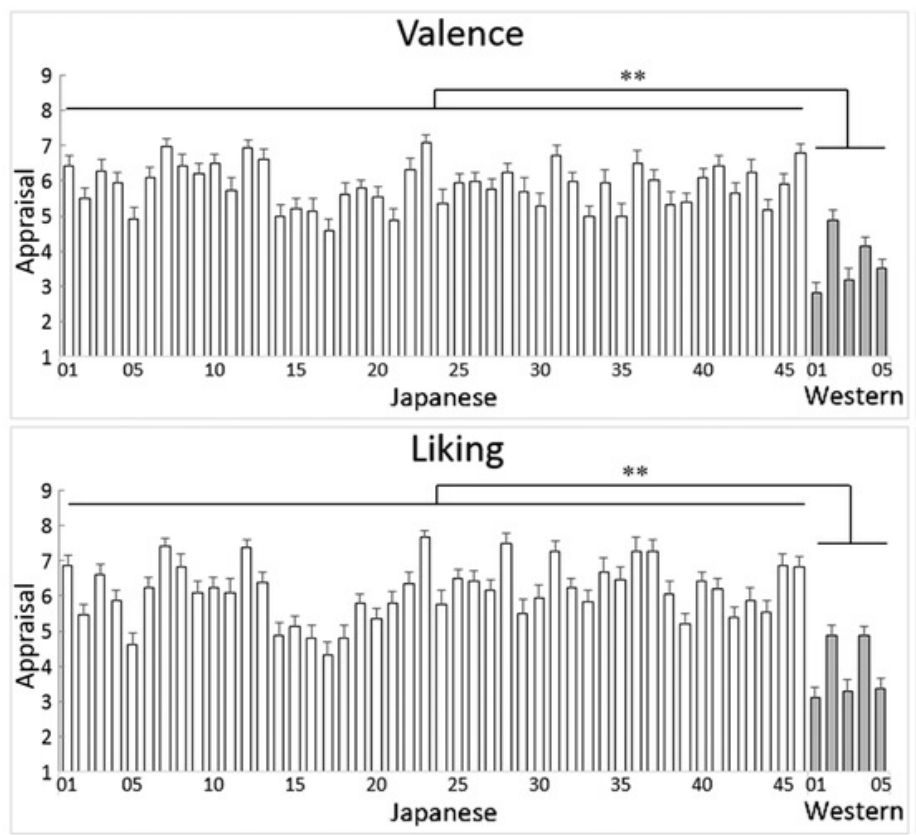

Naturalness

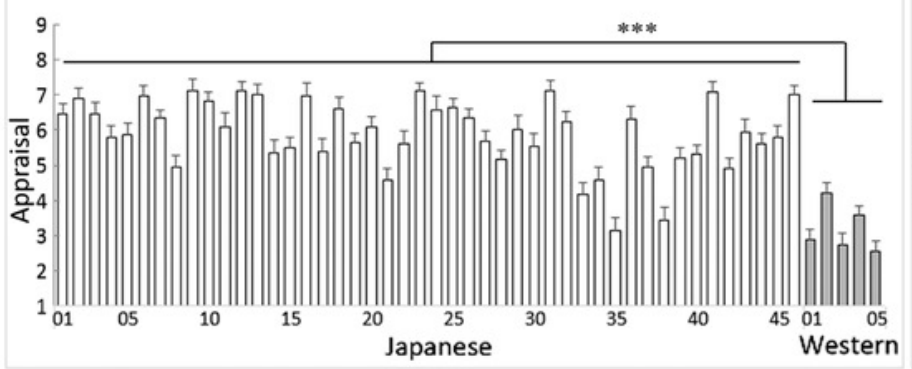

Familiarity

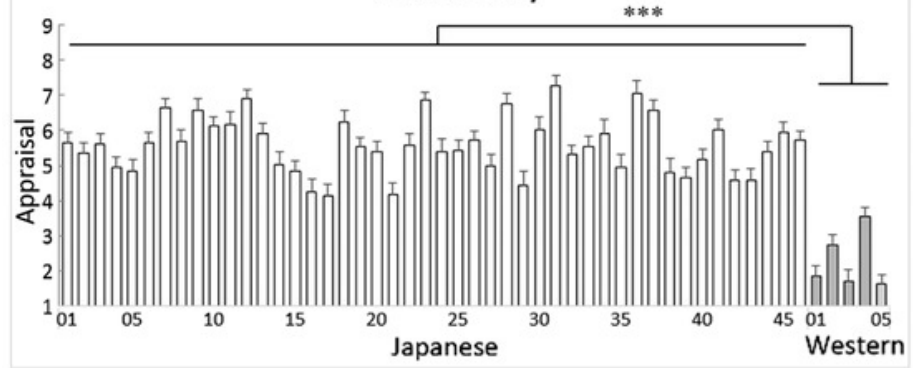

Arousal
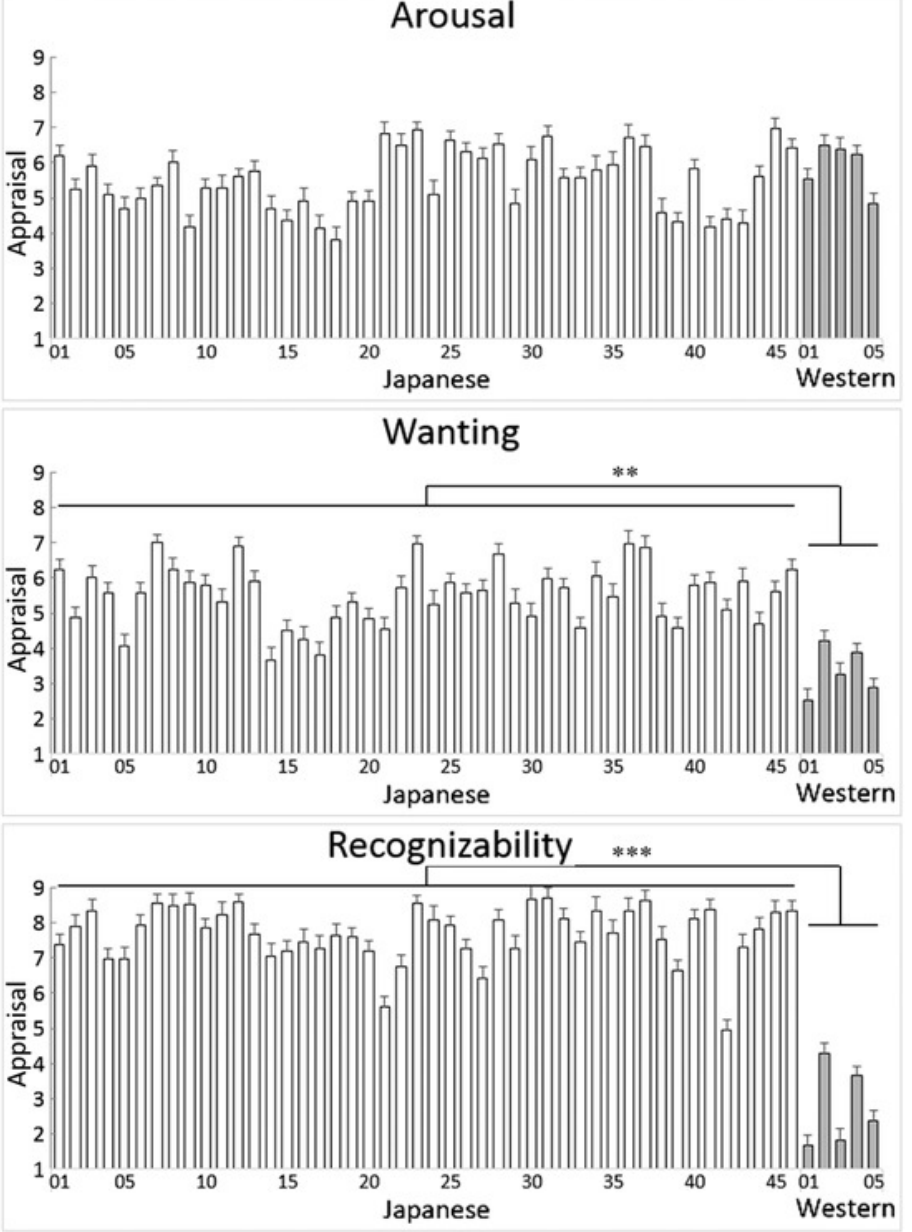
Figure 3

Figure 3. Mean (with standard error) intra-individual correlation coefficients between objective and subjective nutrition information for Japanese food sample images.

\section{Objective - Subjective}

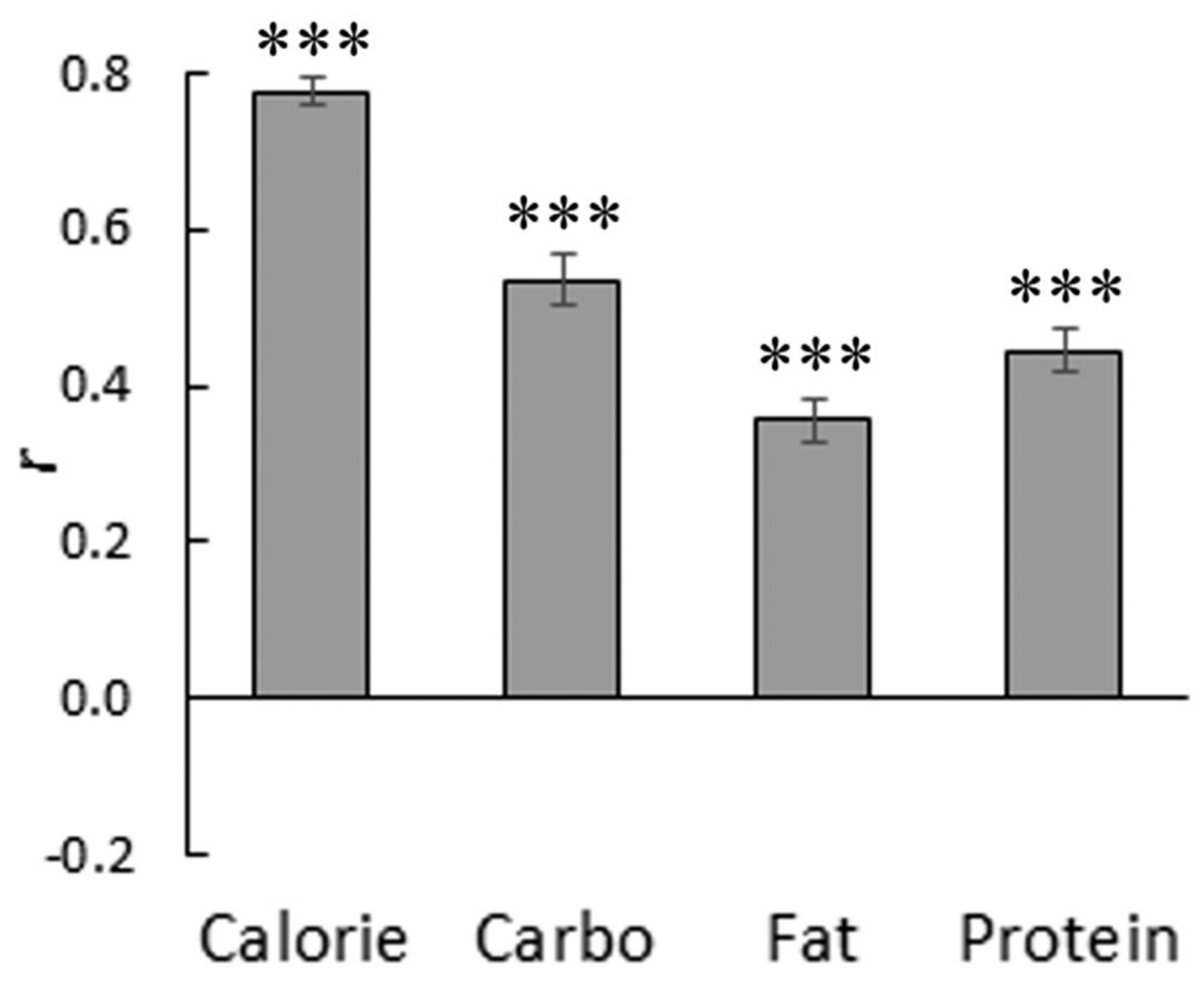


Figure 4

Figure 4. Mean (with standard error) intra-individual correlation coefficients between objective/subjective nutrition information and affective ratings for Japanese food sample images.

The correlations of affective ratings with objective calorie (A), objective carbohydrate (B), objective fat $(C)$, objective protein $(D)$, subjective calorie $(E)$, subjective carbohydrate $(F)$, subjective fat (G), and subjective protein $(\mathrm{H})$ are shown. ${ }^{* * *}, \mathrm{p}<0.001 ;{ }^{* *}, \mathrm{p}<0.01 ; *, \mathrm{p}<$ 0.05 (one-sample t-tests after Fisher's transformation).
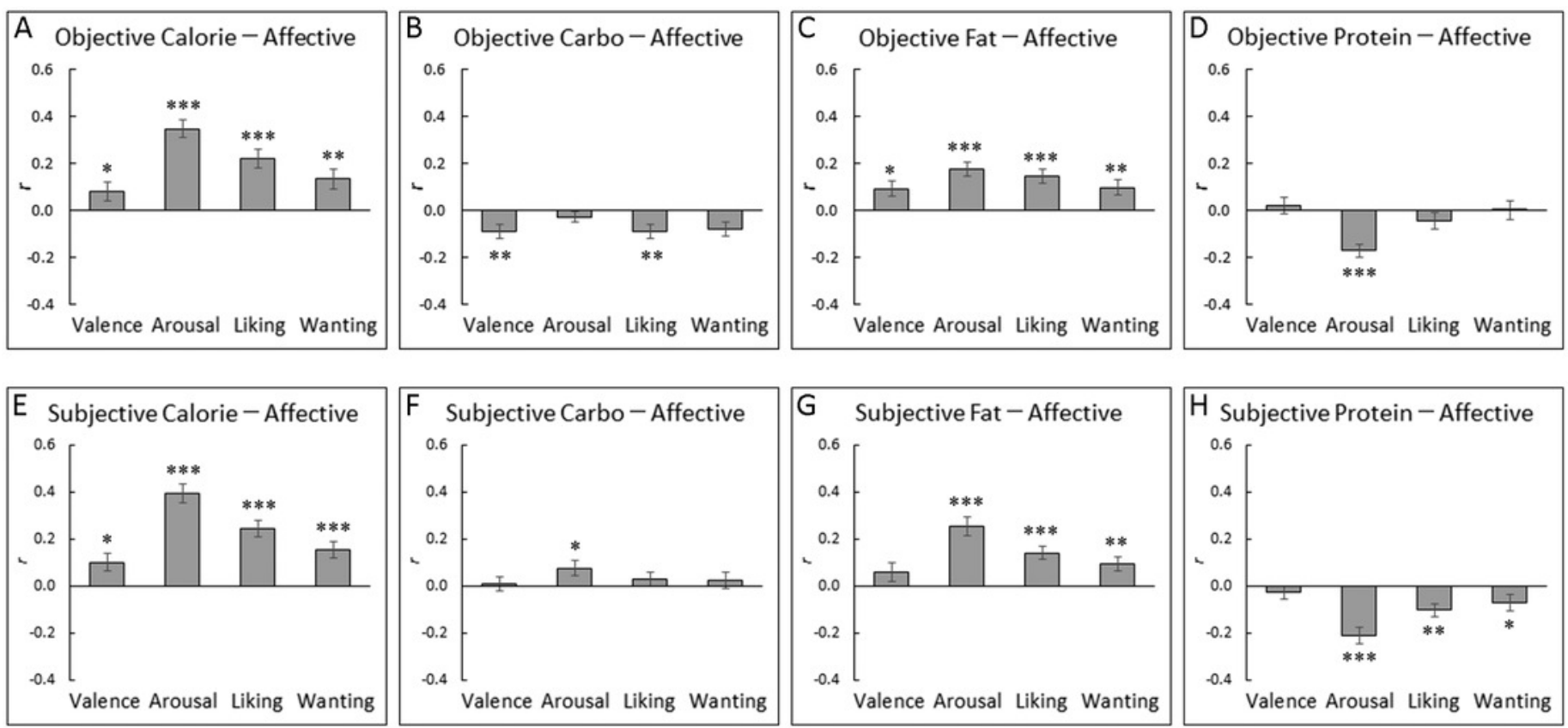